\title{
Exponential decay of correlations for a real-valued dynamical system generated by a $k$ dimensional system
}

\author{
JAGER Lisette, MAES Jules, NINET Alain
}

7 août 2021

\begin{abstract}
We study the real, bounded-variables process $\left\{X_{n}, n \in \mathbb{N}\right\}$ defined by a $k$-term recurrence relation $X_{n+k}=\varphi\left(X_{n}, \ldots, X_{n+k-1}\right)$. We prove the decay of correlations, mainly under purely analytic hypotheses concerning the function $\varphi$ and its partial derivatives.
\end{abstract}

\section{Introduction}

Since the eighties, many statisticians have studied nonlinear time series in order to model various phenomena in Physics, Economics and Finance. About this subject one may consult, for example, Chan-Tong [TON1], Tong [TON2] and Guégan GU]. With chaos theory it became clear that such a series is a perturbed dynamical system. For an extensive survey of litterature about chaos theory, one may read Collet-Eckmann [CE], Lasota-Mackey [LM] or Liverani [LIV]. To undertake the study of nonlinear time series with the help of the theory of dynamical systems, as a first step is the study of a non-perturbed dynamical system defined by a recurrence relation of order $k$ (which Tong names "skeleton" in [TON2]). Indeed, we consider the bounded variables model, $X_{n+k}=\varphi\left(X_{n}, \ldots, X_{n+k-1}\right)$, where $\varphi$ is piecewisely defined on the set $\mathcal{U}^{k}$ and takes its values in $\mathcal{U}$, where $\mathcal{U}=[-L, L]$ for $L \in \mathbb{R}_{+}^{*}$. This model gives rise to a dynamical system $(\Omega, \tau, \mu, T)$ where $\Omega$ is a compact subset of $\mathbb{R}^{k}$ and $\mu$ is a measure preserved by the transformation $T: \Omega \rightarrow \Omega$. Under conditions on $\varphi$, which ensure that $T$ satisfies Saussol's hypotheses [SAU], we obtain the exponential decay of correlations if $T$ is mixing. More precisely, for well-chosen applications $f$ and $g$, we prove that there exist constants $C=C(f, h)>0$ and $\rho \in] 0,1[$ such that :

$$
\left|\int_{\Omega} f \circ T^{n} h d \mu-\int_{\Omega} f d \mu \int_{\Omega} h d \mu\right| \leqslant C \rho^{n} .
$$

This result can be stated in the following way, in the case when $X_{0}$ has distribution $\mu$ :

$$
\left|\operatorname{Cov}\left(f\left(X_{n}\right), h\left(X_{0}\right)\right)\right| \leqslant C \rho^{n} .
$$

Other methods could give the same result, under different hypotheses on the induced system. One can study recurrence times, like Young [YOU] or Gouëzel [GO]. For a general view of all these different techniques, see Alves-Freitas-Luzzato-Vaienti [AFLV].

We had studied the case when $k=2$ in a precedent paper [JMN]. It was then possible to give a very precise result about the localization of the eigenvalues of the $2 \times 2$ matrix (2), which expresses the fact that an auxiliary transformation is expanding. This allows us to obtain better estimates than in the general case, which is the subject of the present work.

At the end of this article, we illustrate our results by applying them to a nonlinear example. 


\section{Hypotheses and results}

Let $L \in \mathbb{R}_{+}^{*}$ and let us consider an application $\varphi:[-L, L]^{k} \rightarrow[-L, L]$, defined piecewisely on $[-L, L]^{k}$.

Under conjugation by an affine function, similar results could be obtained for an application $\varphi$ defined on $[a, b]^{k}$, with values in $[a, b]$.

Suppose that all following conditions are fulfilled :

1. There exists $d \in \mathbb{N}^{*}$ such that

$$
[-L, L]^{k}=\bigcup_{j=1}^{d} O_{j} \cup \mathcal{N}
$$

where the $O_{j}$ are nonempty open subsets, $\mathcal{N}$ is Lebesgue negligible and the union is disjoint. The boundary of each $O_{j}$ is contained in a compact, $C^{1},(k-1)$ - dimensional submanifold of $\mathbb{R}^{k}$.

2. There exists $\varepsilon_{1}>0$ such that, for every $j \in\{1, \ldots, d\}$, there exists a map $\varphi_{j}$ defined on $B_{\varepsilon_{1}}\left(\overline{O_{j}}\right)=\left\{\left(x_{1}, \ldots, x_{k}\right) \in \mathbb{R}^{k}, d\left(\left(x_{1}, \ldots, x_{k}\right), \overline{O_{j}}\right) \leq \varepsilon_{1}\right\}$ with values in $\mathbb{R}$ and satisfying $\left.\varphi_{j}\right|_{O_{j}}=\left.\varphi\right|_{O_{j}}$.

3. The application $\varphi_{j}$ is bounded and $C^{1, \alpha}$ on $B_{\varepsilon_{1}}\left(\overline{O_{j}}\right)$ for an $\left.\left.\alpha \in\right] 0,1\right] 1$, which means that $\varphi_{j}$ is $C^{1}$ and that there exists $C_{j}>0$ such that, for all $\left(u_{1}, \ldots, u_{k}\right),\left(v_{1}, \ldots, v_{k}\right)$ in $B_{\varepsilon_{1}}\left(\overline{O_{j}}\right)$, all $i \in\{1, \ldots, k\}$ :

$$
\left|\frac{\partial \varphi_{j}}{\partial x_{i}}\left(u_{1}, \ldots, u_{k}\right)-\frac{\partial \varphi_{j}}{\partial x_{i}}\left(v_{1}, \ldots, v_{k}\right)\right| \leq C_{j}\left\|\left(u_{1}, \ldots, u_{k}\right)-\left(v_{1}, \ldots, v_{k}\right)\right\|^{\alpha} .
$$

We assume that there exist constants $A>1$ and $\sigma>1$ satisfying $\left.A^{2 / k}>\sigma, M \in\right] 0, M_{0}(\sigma, A)[$ such that for all $2 \leq i \leq k$ :

$\forall\left(u_{1}, \ldots, u_{k}\right) \in B_{\varepsilon_{1}}\left(\overline{O_{j}}\right), \quad\left|\frac{\partial \varphi_{j}}{\partial x_{1}}\left(u_{1}, \ldots, u_{k}\right)\right| \geq A, \quad\left|\frac{\partial \varphi_{j}}{\partial x_{1}}\left(u_{1}, \ldots, u_{k}\right) \times \frac{\partial \varphi_{j}}{\partial x_{i}}\left(u_{1}, \ldots, u_{k}\right)\right| \leq M$

with :

$$
M_{0}(\sigma, A)=\frac{-(k-1) \gamma^{k-1}+\sqrt{(k-1)^{2} \gamma^{2 k-2}+4(k-2) \gamma^{2 k+1}\left(\frac{1}{\gamma^{2}}-\sigma\right)}}{2(k-2) \gamma^{2 k+1}}>0
$$

and

$$
\gamma=A^{-1 / k}
$$

These very tight conditions are due to the loss of precision in the localization of the eigenvalues of the matrix $B$ - see (2) - in the case when $k>2$.

4. The sets $O_{j}$ satisfy the following geometrical condition :2 for all $\left(u_{1}, u_{2}, \ldots, u_{k}\right)$ and $\left(v_{1}, u_{2}, \ldots, u_{k}\right)$ in $B_{\varepsilon_{1}}\left(\overline{O_{j}}\right)$, there exists a $C^{1}$ path $\Gamma=\left(\Gamma_{1}, \ldots, \Gamma_{k}\right):[0,1] \rightarrow B_{\varepsilon_{1}}\left(\overline{O_{j}}\right)$ between $\left(u_{1}, u_{2}, \ldots, u_{k}\right)$ and $\left(v_{1}, u_{2}, \ldots, u_{k}\right)$, with nonzero gradient satisfying

$$
\forall t \in] 0,1\left[,\left|\Gamma_{1}^{\prime}(t)\right|>\frac{M}{A^{2}} \sum_{i=2}^{k}\left|\Gamma_{i}^{\prime}(t)\right| .\right.
$$

1. If $\varphi_{j}$ is $C^{2}$ on $B_{\varepsilon_{1}}\left(\overline{O_{j}}\right)$, it is necessarily $C^{1, \alpha}$ on $B_{\varepsilon_{1}}\left(\overline{O_{j}}\right)$ with $\alpha=1$

2. In favorable cases, the geometrical hypothesis can be replaced by the following one, stronger but much simpler : for all points $\left(u_{1}, u_{2}, \ldots, u_{k}\right)$ and $\left(v_{1}, u_{2}, \ldots, u_{k}\right)$ in $B_{\varepsilon_{1}}\left(\overline{O_{j}}\right)$, the segment $\left[\left(u_{1}, u_{2}, \ldots, u_{k}\right),\left(v_{1}, u_{2}, \ldots, u_{k}\right)\right]$ is contained in $B_{\varepsilon_{1}}\left(\overline{O_{j}}\right)$ 
5. The maximal number of $C^{1} \operatorname{arcs}$ of $\mathcal{N}$ crossing is $Y \in \mathbb{N}^{*}$. Moreover, one sets

$$
s=\frac{1}{\sqrt{\sigma}}<1
$$

and imposes that

$$
\eta:=s^{\alpha}+\frac{4 s}{1-s} Y \frac{\gamma_{k-1}}{\gamma_{k}}<1
$$

where $\gamma_{k}=\frac{\pi^{k / 2}}{\Gamma\left(\frac{k}{2}+1\right)}$ is the volume of the unit sphere of $\mathbb{R}^{k}$.

(This last condition is the most restricting of all, for it gives an upper bound for $s$ and thus a lower bound for $\sigma$ and $A$.)

For every $j \in\{1, \ldots, d\}$, one denotes by $U_{j}$ (resp. $\left.W_{j}, \mathcal{N}^{\prime}\right)$ the image of $O_{j}\left(\right.$ resp. $\left.B_{\varepsilon_{1}}\left(\overline{O_{j}}\right), \mathcal{N}\right)$ under the transformation which associates with $\left(u_{1}, \ldots, u_{k}\right) \in \mathbb{R}^{k}$ the point $\left(u_{1}, \gamma u_{2}, \ldots, \gamma^{k-1} u_{k}\right)$. The set $\Omega=[-L, L] \times[-\gamma L, \gamma L] \times \ldots \times\left[-\gamma^{k-1} L, \gamma^{k-1} L\right]$, with which we shall work, is the image of $[-L, L]^{k}$ under the same transformation.

For every non-negligible Borel set $S$ of $\mathbb{R}^{k}$, for every $f \in L_{m}^{1}\left(\mathbb{R}^{k}, \mathbb{R}\right)$, one sets :

$$
O s c(f, S)=\underset{S}{\operatorname{Esupf}}-\underset{S}{\operatorname{Einf} f}
$$

where Esup et Einf are the essential supremum and infimum on $S$ with respect to the Lebesgue measure $\stackrel{S}{m}$.

One then defines the norm $\|\cdot\|_{\alpha}$ by

$$
|f|_{\alpha}=\sup _{0<\varepsilon<\varepsilon_{1}} \varepsilon^{-\alpha} \int_{\mathbb{R}^{k}} \operatorname{Osc}\left(f, B_{\varepsilon}\left(x_{1}, \ldots, x_{k}\right)\right) d x_{1} \ldots d x_{k}, \quad\|f\|_{\alpha}=\|f\|_{L_{m}^{1}}+|f|_{\alpha}
$$

and the set $V_{\alpha}=\left\{f \in L_{m}^{1}\left(\mathbb{R}^{k}, \mathbb{R}\right),\|f\|_{\alpha}<+\infty\right\}$.

We introduce similar notions on $\Omega$ : for every $0<\varepsilon_{0}<\gamma^{k-1} L$, for every $g \in L_{m}^{\infty}(\Omega, \mathbb{R})$, we define :

$$
N(g, \alpha, L)=\sup _{0<\varepsilon<\varepsilon_{0}} \varepsilon^{-\alpha} \int_{\Omega} \operatorname{Osc}\left(g, B_{\varepsilon}\left(x_{1}, \ldots, x_{k}\right) \cap \Omega\right) d x_{1} \ldots d x_{k} .
$$

One then sets :

$$
\|g\|_{\alpha, L}=N(g, \alpha, L)+2 K(\Omega) \varepsilon_{0}^{1-\alpha}\|g\|_{\infty}+\|g\|_{L_{m}^{1}}
$$

where $K(\Omega)=2^{k+2}\left(\sum_{i=1}^{k} 2 \gamma^{i-1} L\right)^{k-1}=2^{2 k+1} L^{k-1}\left(\frac{1-\gamma^{k}}{1-\gamma}\right)^{k-1}$.

The function $g$ is said to belong to $V_{\alpha}(\Omega)$ if this expression is finite. This set does not depend on the choice of $\varepsilon_{0}$, but $N$ and $\|\cdot\|_{\alpha, L}$ do.

There exist relations between the sets $V_{\alpha}(\Omega)$ and $V_{\alpha}$. Indeed, one can prove the following result using Proposition 3.4 of [SAU] :

\section{Proposition 1}

1. If $g \in V_{\alpha}(\Omega)$ and if one extends $g$ to a function $f$ defined on $\mathbb{R}^{k}$, setting $f(x)=0$ if $x \notin \Omega$, then $f \in V_{\alpha}$ and

$$
\|f\|_{\alpha} \leq\|g\|_{\alpha, L} .
$$

2. Conversely let $f \in V_{\alpha}$ and set $g=f \mathbf{1}_{\Omega}$. Then $g \in V_{\alpha}(\Omega)$ and the following holds :

$$
\|g\|_{\alpha, L} \leq\left(1+2 K(\Omega) \frac{\max \left(1, \varepsilon_{0}^{\alpha}\right)}{\gamma_{k} \varepsilon_{0}^{k-1+\alpha}}\right)\|f\|_{\alpha} .
$$


Under the hypotheses 1-5 listed above, we obtain a first result :

Theorem 2 Let $T$ be the transformation defined on $\Omega$ by $: \forall u=\left(u_{1}, \ldots, u_{k}\right) \in U_{j}$ :

$$
T(u)=T_{j}(u)=\left(\frac{u_{2}}{\gamma}, \ldots, \frac{u_{k}}{\gamma}, \gamma^{k-1} \varphi_{j}\left(u_{1}, \frac{u_{2}}{\gamma}, \ldots, \frac{u_{k}}{\gamma^{k-1}}\right)\right) .
$$

The applications $T_{j}$ can be defined naturally on $W_{j}$ by the same formula. Then

1. The Frobenius-Perron operator $P: L_{m}^{1}(\Omega) \rightarrow L_{m}^{1}(\Omega)$ associated with $T$ has a finite number of eigenvalues of modulus $1, \lambda_{1}, \ldots, \lambda_{r}$.

2. For every $i \in\{1, \ldots, r\}$, the eigenspace $E_{i}=\left\{f \in L_{m}^{1}(\Omega): P f=\lambda_{i} f\right\}$ associated with the eigenvalue $\lambda_{i}$ is finite-dimensional and contained in $V_{\alpha}(\Omega)$.

3. The operator $P$ decomposes as

$$
P=\sum_{i=1}^{r} \lambda_{i} P_{i}+Q
$$

where the $P_{i}$ are projections on the spaces $E_{i},\left\|P_{i}\right\|_{1} \leq 1$ and $Q$ is a linear operator defined on $L_{m}^{1}(\Omega)$, such that $Q\left(V_{\alpha}(\Omega)\right) \subset V_{\alpha}(\Omega)$, $\sup _{n \in \mathbb{N}^{*}}\left\|Q^{n}\right\|_{1}<\infty$ and $\left\|Q^{n}\right\|_{\alpha, L}=O\left(q^{n}\right)$ when $n \rightarrow+\infty$, for a given $q \in] 0,1\left[\right.$. Moreover, $P_{i} P_{j}=0$ if $i \neq j, P_{i} Q=Q P_{i}=0$ for every $i$.

4. The operator $P$ has the eigenvalue 1 . Set $\lambda_{1}=1$, let $h_{*}=P_{1} \mathbf{1}_{\Omega}$ and $d \mu=h_{*} d m$. Then $\mu$ is the greatest absolutely continuous invariant measure (ACIM) of $T$, which means that, if $\nu<<m$ and if $\nu$ is T-invariant, then $\nu<<\mu$.

5. The support of $\mu$ can be decomposed into a finite number of mutually disjoint measurable sets, on which a power of $T$ is mixing. More precisely, for every $j \in\left\{1,2, \ldots, \operatorname{dim}\left(E_{1}\right)\right\}$, there exist a number $L_{j} \in \mathbb{N}^{*}$ and $L_{j}$ mutually disjoint sets $W_{j, l}\left(0 \leq l \leq L_{j}-1\right)$, satisfying $T\left(W_{j, l}\right)=W_{j, l+1} \bmod \left(L_{j}\right), T^{L_{j}}$ being mixing on every $W_{j, l}$. One denotes by $\mu_{j, l}$ the normalized restriction of $\mu$ on $W_{j, l}$, defined by

$$
\mu_{j, l}(B)=\frac{\mu\left(B \cap W_{j, l}\right)}{\mu\left(W_{j, l}\right)}, d \mu_{j, l}=\frac{h^{*} \mathbf{1}_{W_{j, l}}}{\mu\left(W_{j, l}\right)} d m .
$$

Saying that $T^{L_{j}}$ is mixing on every $W_{j, l}$ means that, for every $f \in L_{\mu_{j, l}}^{1}\left(W_{j, l}\right)$ and every $h \in L_{\mu_{j, l}}^{\infty}\left(W_{j, l}\right)$,

$$
\lim _{n \rightarrow+\infty}<T^{n L_{j}} f, h>_{\mu_{j, l}}=<f, 1>_{\mu_{j, l}}<1, h>_{\mu_{j, l}}
$$

with indifferently used notations : $<f, g>_{\mu^{\prime}}=\mu^{\prime}(f g)=\int f g d \mu^{\prime}$.

6. Moreover, there exist real constants $C>0$ and $0<\rho<1$ such that, for every $h$ in $V_{\alpha}(\Omega)$ and $f \in L_{\mu}^{1}(\Omega)$, the following holds :

$$
\left|\int_{\Omega} f \circ T^{n \times p p c m\left(L_{i}\right)} h d \mu-\sum_{j=1}^{\operatorname{dim}\left(E_{1}\right)} \sum_{l=0}^{L_{j}-1} \mu\left(W_{j, l}\right)<f, 1>_{\mu_{j, l}}<1, h>_{\mu_{j, l}}\right| \leq C\|h\|\left\|_{\alpha, \Omega}\right\| f \|_{L_{\mu}^{1}(\Omega)} \rho^{n} .
$$

7. If, moreover, $T$ is mixing, 3 the preceding result can be stated as : there exist real constants $C>0$ and $0<\rho<1$ such that, for every $h$ in $V_{\alpha}(\Omega)$ and $f \in L_{\mu}^{1}(\Omega)$, one has :

$$
\left|\int_{\Omega} f \circ T^{n} h d \mu-\int_{\Omega} f d \mu \int_{\Omega} h d \mu\right| \leq C|| h\left\|_{\alpha, \Omega}\right\| f \|_{L_{\mu}^{1}(\Omega)} \rho^{n} .
$$

\footnotetext{
3. Which is equivalent to : if 1 is the only modulus- 1 eigenvalue of $P$ and if, additionnaly, it is simple
} 
Let us now come back to the initial system and let us try to deduce the invariant law associated with $X_{n}$. If the sequence $\left(X_{n}\right)_{n}$ is defined by the initial terms $X_{0}, \ldots, X_{k-1}$, with values in $[-L, L]$, and the recurrence relation $X_{n+k}=\varphi\left(X_{n}, \ldots, X_{n+k-1}\right)$, one sets $Z_{n}=$ $\left(\gamma^{j-1} X_{n+j-1}\right)_{1 \leq j \leq k}$. Then $\left(Z_{n}\right)_{n}$ satisfies the recurrence relation $Z_{n+1}=T\left(Z_{n}\right)$, which yields the following result :

Theorem 3 If the random variable $Z_{0}=\left(\gamma^{j-1} X_{j-1}\right)_{1 \leq j \leq k}$ has density $h_{*}$, then, for every $n \geq 0$, $Z_{n}$ has density $h_{*}$. Computing the marginal distributions, we get as a consequence that for every $n \in \mathbb{N}, X_{n}$ has a density $h_{i n v}$ which has the following expressions : for every $j \in\{0, \ldots, k-1\}$

$$
\forall u \in[-L, L], \quad h_{i n v}(u)=\gamma^{j} \int_{\mathbb{R}^{k-1}} h_{*}\left(z_{1}, \ldots, \gamma^{j} u, \ldots, z_{k}\right) d \check{z}_{j+1}
$$

where $d \check{z}_{j+1}$ means that one integrates with respect to all coordinates of $z$ but $z_{j+1}$.

Indeed, $\gamma^{j} X_{n}$ is the $(j+1)$-th coordinate of $Z_{n-j}$ if $j=0, \ldots, k-1$. Let us consider a Borel set $A$ of $\mathbb{R}$. Then, for $j \in\{0, \ldots, k-1\}$,

$$
\begin{aligned}
P\left(X_{n} \in A\right) & =P\left(Z_{n-j} \in \mathbb{R}^{j} \times \gamma^{j} A \times \mathbb{R}^{k-j-1}\right) \\
& =\int_{\mathbb{R}^{j} \times \gamma^{j} A \times \mathbb{R}^{k-j-1}} h_{*}\left(z_{1}, \ldots, z_{k}\right) d z_{1} \ldots d z_{k} \\
& =\int_{\mathbb{R}^{j} \times A \times \mathbb{R}^{k-j-1}} h_{*}\left(z_{1}, \ldots, \gamma^{j} u, \ldots, z_{k}\right) d \check{z}_{j+1} \gamma^{j} d u \quad \text { with } z_{j+1}=\gamma^{j} u \\
& =\int_{A}\left(\int_{\mathbb{R}^{k-1}} h_{*}\left(z_{1}, \ldots, \gamma^{j} u, \ldots, z_{k}\right) d \check{z}_{j+1}\right) \gamma^{j} d u,
\end{aligned}
$$

which gives the desired result.

If $F$ is defined on $[-L, L]$ and if $s \in\{1, \ldots, k\}$, let us denote by $T_{s} F$ the function defined on $\Omega$ by

$$
T_{s} F(z)=T_{s} F\left(z_{1}, \ldots, z_{k}\right)=F\left(z_{s} \gamma^{1-s}\right) .
$$

The following Lemma is then a direct consequence of point 6 in Theorem 2, applied to $T_{s} F$ and $T_{r} H$ for $s, r \in\{1, \ldots, k\}$ :

Lemma 4 For every Borel set $B$ of $[-L, L]$ and every interval $I$ of $[-L, L]$, if $Z_{0}$ has the invariant distribution, one has :

$$
\begin{aligned}
& \left|P\left(X_{n \times p p c m\left(L_{i}\right)+s-1} \in B, X_{r-1} \in I\right)-\sum_{j=1}^{\operatorname{dim}\left(E_{1}\right)} \sum_{l=0}^{L_{j}-1} \mu\left(W_{j, l}\right)<T_{s} \mathbf{1}_{B}, 1>_{\mu_{j, l}}<1, T_{r} \mathbf{1}_{I}>_{\mu_{j, l}}\right| \\
& \quad \leq\left((2 L)^{k} \gamma^{k(k-1) / 2}+4(2 L)^{k-1} \gamma^{1-r+k(k-1) / 2} \varepsilon_{0}^{1-\alpha}+2^{2 k} L^{k-1}\left(\frac{1-\gamma^{k}}{1-\gamma}\right)^{k-1} \varepsilon_{0}^{1-\alpha}\right) C \rho^{n} .
\end{aligned}
$$

More generally, let $F$, defined and measurable on $[-L, L]$, be such that $T_{s} F$ belongs to $L_{\mu}^{1}(\Omega)$. Let $H \in L_{m}^{\infty}([-L, L])$ be such that $\sup _{0<\delta<\varepsilon_{0} \gamma^{1-r}} \delta^{-\alpha} \int_{[-L, L]} \operatorname{Osc}(H] x-,\delta, x+\delta[\cap[-L, L]) d x<+\infty$. Then $T_{r} H \in V_{\alpha}(\Omega)$ and

$$
\left|E\left(F\left(X_{n \times p p c m\left(L_{i}\right)+s-1}\right) H\left(X_{r-1}\right)\right)-\sum_{j=1}^{\operatorname{dim}\left(E_{1}\right)} \sum_{l=0}^{L_{j}-1} \mu\left(W_{j, l}\right) \mu_{j, l}\left(T_{s} F\right) \mu_{j, l}\left(T_{r} H\right)\right| \leq C(F, H, L) \rho^{n}
$$


with

$$
\begin{aligned}
& C(F, H, L)=C\left\|T_{s} F\right\|_{L_{\mu}^{1}}\left((2 L)^{k-1} \gamma^{k(k-1) / 2}\|H\|_{L_{m}^{1}([-L, L])}\right. \\
& +(2 L)^{k-1} \gamma^{(k(k-1) / 2)-\alpha(r-1)} \sup _{0<\delta<\varepsilon_{0} \gamma^{1-r}} \delta^{-\alpha} \int_{[-L, L]} \operatorname{Osc}(H,] x-\delta, x+\delta[\cap[-L, L]) d x \\
& \left.+2^{2 k} L^{k-1}\left(\frac{1-\gamma^{k}}{1-\gamma}\right)^{k-1} \varepsilon_{0}^{1-\alpha}\|H\|_{L_{m}^{\infty}([-L, L])}\right) .
\end{aligned}
$$

This last result, which gives the exponential decay of correlations, is a straightforward consequence of Lemma 4 and of the remark in point 7 , Theorem 2 .

Theorem 5 If, moreover, $T$ is mixing, then

$$
\left|\operatorname{Cov}\left(F\left(X_{n+s-1}\right), H\left(X_{r-1}\right)\right)\right| \leq C(F, H, L) \rho^{n} .
$$

\section{Proofs}

Theorem 2 is a consequence of Theorems 5.1 and 6.1 of [SAU], which rely on [ITM], as well as [HK] in the case when $d=1$, where the use of bounded-variation functions is possible. The difficulty lies in verifying that $T$ satisfies Hypotheses (PE1) to (PE5).

To prove that (PE2) is satisfied, we shall first establish that $T_{j}$ is a $C^{1}$ diffeomorphism on $W_{j}$ onto $T_{j}\left(W_{j}\right)$. Hypothesis 3 about $\frac{\partial \varphi_{j}}{\partial x_{1}}$ assures that $T_{j}$ is a local diffeomorphism. To check that it is injective, let us consider two different points $u$ and $v$ of $W_{j}$, such that $T_{j}(u)=T_{j}(v)$. Then $u_{i}=v_{i}$ for every $2 \leq i \leq k$ and

$$
\varphi_{j}\left(u_{1}, \frac{u_{2}}{\gamma}, \ldots, \frac{u_{k}}{\gamma^{k-1}}\right)=\varphi_{j}\left(v_{1}, \frac{u_{2}}{\gamma}, \ldots, \frac{u_{k}}{\gamma^{k-1}}\right) .
$$

Using the geometrical hypothesis 4 and applying the fundamental theorem of calculus to $t \mapsto$ $\varphi_{j}(\Gamma(t))$ leads to a contradiction. The regularity hypotheses on the $\varphi_{j}$ (and hence on the $T_{j}$ ) allow to prove that $\operatorname{det}\left(D T_{j}^{-1}\right)$ is $\alpha$-Hölder, provided the domain is conveniently restricted. One can see that there exist, for each $\beta_{j}>0$, an open and relatively compact set $\mathcal{V}_{j}$ and a real constant $c_{j}$ such that the following holds

$-\overline{U_{j}} \subset \mathcal{V}_{j} \subset \overline{\mathcal{V}_{j}} \subset W_{j}$;

$-B_{\beta_{j}}\left(T_{j}\left(U_{j}\right)\right) \subset T_{j}\left(\mathcal{V}_{j}\right)$

- for every $\varepsilon<\beta_{j}$, every $z \in T_{j}\left(\mathcal{V}_{j}\right)$ and all $x, y \in B_{\varepsilon}(z) \cap T_{j}\left(\mathcal{V}_{j}\right)$,

$$
\left|\operatorname{det}\left(D T_{j}^{-1}(x)\right)-\operatorname{det}\left(D T_{j}^{-1}(y)\right)\right| \leq c_{j}\left|\operatorname{det}\left(D T_{j}^{-1}(z)\right)\right| \varepsilon^{\alpha} .
$$

Setting $\beta=\min _{j} \beta_{j}>0$ and $c=\max _{j} c_{j}>0$, one obtain constants which are convenient for every $j \in\{1, \ldots, d\}$. Hence (PE2) is satisfied.

This allows us to specify the open sets on which we shall work. There exists $\varepsilon_{2}>0$ such that, for every $j \in\{1, \ldots, d\}, B_{2 \varepsilon_{2}}\left(\overline{U_{j}}\right) \subset \mathcal{V}_{j} \subset W_{j}$. From now on, one sets $V_{j}=B_{\varepsilon_{2}}\left(\overline{U_{j}}\right)$. Then $T_{j}\left(V_{j}\right)$ is open and $T_{j}\left(\overline{U_{j}}\right)$ is compact and contained in $T_{j}\left(V_{j}\right)$. One can find a positive $\varepsilon_{0,1}$ such that $B_{\varepsilon_{0,1}}\left(T_{j}\left(\overline{U_{j}}\right)\right) \subset T_{j}\left(V_{j}\right)$ for every $j$, which proves that Hypothesis (PE1) is satisfied.

Hypothesis (PE3) is clearly fulfilled since $\Omega=\bigcup_{j=1}^{d} U_{j} \cup \mathcal{N}^{\prime}$ is a disjoint union of open sets and of a negligible set. 
For (PE4), we need two steps. We first prove that the map is locally expanding (when the preimages in $\mathcal{V}_{j}$ are sufficiently near, Proposition 6). Then we prove the hypothesis itself (Proposition (7), in the case when the images in $T_{j}\left(V_{j}\right)$ are sufficiently near.

Proposition 6 Let $u$ and $v \in \mathcal{V}_{j}$ be such that the segment $[u, v]$ is contained in $\mathcal{V}_{j}$. Then

$$
\left\|T_{j}(u)-T_{j}(v)\right\|^{2} \geq \frac{1}{s^{2}}\|u-v\|^{2} .
$$

Proof : One applies the fundamental theorem of calculus to the map defined on $[0,1]$ by $t \mapsto$ $\varphi_{j}\left(v_{1}+t\left(u_{1}-v_{1}\right), \frac{v_{2}+t\left(u_{2}-v_{2}\right)}{\gamma}, \ldots, \frac{v_{k}+t\left(u_{k}-v_{k}\right)}{\gamma^{k-1}}\right)$, which yields a $\left.c \in\right] 0,1[$ such that

$$
\left\|T_{j}(u)-T_{j}(v)\right\|^{2}=\left(v_{1}-u_{1}, \ldots, v_{k}-u_{k}\right) B\left(\begin{array}{l}
v_{1}-u_{1} \\
\vdots \\
v_{k}-u_{k}
\end{array}\right)
$$

where $B=\left(b_{i, l}\right)_{1 \leq i, l \leq k}$ is the matrix with coefficients

$$
\left\{\begin{aligned}
b_{i, l} & =\gamma^{2 k-i-l} \frac{\partial \varphi_{j}}{\partial x_{i}}\left(M_{c}\right) \frac{\partial \varphi_{j}}{\partial x_{l}}\left(M_{c}\right) \quad \text { if } i \neq l \\
b_{1,1} & =\gamma^{2 k-2}\left(\frac{\partial \varphi_{j}}{\partial x_{1}}\left(M_{c}\right)\right)^{2}, \\
b_{i, i} & =\frac{1}{\gamma^{2}}+\gamma^{2(k-i)}\left(\frac{\partial \varphi_{j}}{\partial x_{i}}\left(M_{c}\right)\right)^{2} \text { if } i>1
\end{aligned}\right.
$$

with $M_{c}=\left(v_{1}+c\left(u_{1}-v_{1}\right), \frac{v_{2}+c\left(u_{2}-v_{2}\right)}{\gamma}, \ldots, \frac{v_{k}+c\left(u_{k}-v_{k}\right)}{\gamma^{k-1}}\right)$.

The matrix $B$ is real and symmetrical. Its eigenvalues are contained in the Gershgörin disks and hence in the following domain

$$
\bigcup_{i=1}^{k}\left[b_{i, i}-\sum_{l \neq i}\left|b_{i, l}\right|, b_{i, i}+\sum_{l \neq i}\left|b_{i, l}\right|\right] .
$$

We shall establish that all these intervals are contained in $[\sigma,+\infty[$. To that aim, it is sufficient to prove that $b_{i, i}-\sum_{l \neq i}\left|b_{i, l}\right| \geq \sigma$ for every $i$.

According to Hypothesis 3 one has, for every $l>1$, $\left|\frac{\partial \varphi_{j}}{\partial x_{l}}\left(M_{c}\right)\right| \leq \frac{M}{A}$, which implies that

$$
\left\{\begin{array}{l}
b_{1,1}-\sum_{l \neq 1}\left|b_{1, l}\right| \geq \gamma^{2 k-2} A^{2}-M \sum_{l \neq 1} \gamma^{2 k-1-l} \\
b_{i, i}-\sum_{l \neq i}\left|b_{i, l}\right| \geq \frac{1}{\gamma^{2}}-M \gamma^{2 k-i-1}-\frac{M^{2}}{A^{2}} \sum_{l \neq i, l>1} \gamma^{2 k-i-l} \quad \text { for } i>1
\end{array}\right.
$$

Since $\gamma<1$ and $2 k-1-l \geq k-1$ one eventually gets :

$$
\left\{\begin{array}{l}
b_{1,1}-\sum_{l \neq 1}\left|b_{1, l}\right| \geq \gamma^{2 k-2} A^{2}-M(k-1) \gamma^{k-1} \\
b_{i, i}-\sum_{l \neq i}\left|b_{i, l}\right| \geq \frac{1}{\gamma^{2}}-M \gamma^{k-1}-\frac{M^{2}}{A^{2}}(k-2) \gamma \quad \text { for } i>1 .
\end{array}\right.
$$


Since $\gamma=A^{-1 / k}$, one derives the inequalities :

$$
\left\{\begin{array}{l}
b_{1,1}-\sum_{l \neq 1}\left|b_{1, l}\right| \geq \frac{1}{\gamma^{2}}-M(k-1) \gamma^{k-1} \\
b_{i, i}-\sum_{l \neq i}\left|b_{i, l}\right| \geq \frac{1}{\gamma^{2}}-M(k-1) \gamma^{k-1}-M^{2}(k-2) \gamma^{2 k+1} \quad \text { for } i>1
\end{array}\right.
$$

Therefore, the eigenvalues of $B$ are all greater than or equal to $\frac{1}{\gamma^{2}}-M(k-1) \gamma^{k-1}-M^{2}(k-$ 2) $\gamma^{2 k+1}$. Since $\left.M \in\right] 0, M_{0}(\sigma, A)[$, one has :

$$
\frac{1}{\gamma^{2}}-M(k-1) \gamma^{k-1}-M^{2}(k-2) \gamma^{2 k+1} \geq \sigma .
$$

Consequently, the eigenvalues of $B$ are all greater than or equal to $\sigma=\frac{1}{s^{2}}$, which gives the desired result. Notice that this last inequality compells us to choose $\frac{1}{\gamma^{2}}>\sigma$.

Compacity arguments give the existence of $\varepsilon_{0,2}>0$ such that, for every $u \in \overline{V_{j}}$,

$$
B_{\varepsilon_{0,2}}\left(T_{j}(u)\right) \subset T_{j}\left(B_{\varepsilon_{2}}(u)\right) .
$$

Proposition 7 Let $\varepsilon_{0}=\min \left(\varepsilon_{0,1}, \varepsilon_{0,2}\right)>0$. Recall that $\overline{U_{j}} \subset V_{j} \subset \overline{V_{j}} \subset \mathcal{V}_{j} \subset W_{j}$. As a consequence,

- for all $x, y \in T_{j}\left(V_{j}\right)$ satisfying $\|x-y\|<\varepsilon_{0}$, the following inequality is valid:

$$
s^{2}\|x-y\|>\left\|T_{j}^{-1}(x), T_{j}^{-1}(y)\right\| .
$$

$-B_{\varepsilon_{0}}\left(T_{j}\left(\overline{U_{j}}\right)\right) \subset T_{j}\left(V_{j}\right)$.

Proof: The second statement comes from the fact that $\varepsilon_{0} \leq \varepsilon_{0,1}$ and from the results we obtained in relation with (PE1).

Let us prove the first statement, which implies Condition (PE4) of Saussol. Let $x, y \in T_{j}\left(V_{j}\right)$ satisfy $\|x-y\|<\varepsilon_{0}$. Set $u=T_{j}^{-1}(x) \in V_{j}$. According to the preceding remark, as $\varepsilon_{0}$ is smaller than $\varepsilon_{0,2}$,

$$
y \in B_{\varepsilon_{0}}\left(T_{j}(u)\right) \subset T_{j}\left(B_{\varepsilon_{2}}(u)\right) .
$$

Hence $v=T_{j}^{-1}(y) \in B_{\varepsilon_{2}}(u) \subset \mathcal{V}_{j}$. According to Proposition 6 ,

$$
\|x-y\|^{2}=\left\|T_{j}(u)-T_{j}(v)\right\|^{2}>\sigma\|u-v\|^{2},
$$

which proves the result.

To conclude, Hypothesis (PE5) is a consequence of Lemma 2.1 of Saussol and of Hypothesis 5 .

Since the hypotheses (PE1) to (PE5) are satisfied, Theorem 5.1 of [SAU] implies the properties 1 to 5 of Theorem 2 about $V_{\alpha}$ and $L_{m}^{1}$. Now, if $f \in E_{i}, f$ is equal to zero on $\Omega^{c}$, which implies that $f \in L_{m}^{1}(\Omega)$ and $V_{\alpha}(\Omega)$.

To prove point 6 , we shall apply Theorem 6.1 of [SAU] on every subset $W_{j, l}$, on which a suitable power of $T$ is mixing. Adopting the notations of Point 5 of Theorem 5.2 of [SAU], there exist real constants $C>0$ and $\rho \in] 0,1$ [ such that, for every $(j, l)$ satisfying $1 \leq j \leq \operatorname{dim}\left(E_{1}\right)$, $0 \leq l \leq L_{j}-1$, every function $f \in L_{\mu_{j, l}}^{1}(\Omega)$ and every function $h \in V_{\alpha}(\Omega)$,

$$
\left.\left|\int_{\Omega}\left(f-\mu_{j, l}(f)\right) \circ T^{n L_{j}} h d \mu_{j, l}\right| \leq C|| f-\mu_{j, l}(f)\right)\left\|_{L_{\mu_{j, l}^{1}}^{1}}\right\| h \|_{\alpha, L} \rho^{n} .
$$


Let us choose, then, $h \in V_{\alpha}(\Omega)$ and $f \in L_{\mu}^{1}(\Omega)$ (with the result that $f \in L_{\mu_{j, l}}^{1}(\Omega)$ for every $j, l$ ). Taking the smallest common multiple $L^{\prime}$ of the $L_{j}$ and summing the above inequalities, with $n$ replaced with $n \frac{L^{\prime}}{L_{j}}$, we obtain that

$$
\left|\int_{\Omega} f \circ T^{n L^{\prime}} h d \mu-\sum_{j=1}^{\operatorname{dim}\left(E_{1}\right)} \sum_{l=0}^{L_{j}-1} \mu\left(W_{j, l}\right) \mu_{j, l}(f) \mu_{j, l}(h)\right| \leq C\|h\|_{\alpha, L}\|f\|_{L_{\mu}^{1}} \rho^{n},
$$

remarking that $\left.\| f-\mu_{j, l}(f)\right)\left\|_{L_{\mu_{j, l}}^{1}} \leq 2\right\| f \|_{L_{\mu}^{1}}$.

Point 7 is a straightforward consequence of Point 6 , since $\operatorname{dim}\left(E_{1}\right)=1$ and $L_{1}=1$. This concludes the proof of Theorem 2

Let us now turn to Lemma 4. If $Z_{0}=\left(X_{0}, \ldots, \gamma^{k-1} X_{k-1}\right)$ has distribution $\mu$, then this is the case for $Z_{n}=\left(X_{n}, \ldots, \gamma^{k-1} X_{n+k-1}\right)$ as well. If $f \in L_{\mu}^{1}(\Omega)$ and if $h \in V_{\alpha}(\Omega)$, one has :

$$
\left|E\left(f\left(Z_{n L^{\prime}}\right) h\left(Z_{0}\right)\right)-\sum_{j=1}^{\operatorname{dim}\left(E_{1}\right)} \sum_{l=0}^{L_{j}-1} \mu\left(W_{j, l}\right) \mu_{j, l}(f) \mu_{j, l}(h)\right| \leq C\|f\|_{L_{\mu}^{1}}\|h\|_{\alpha, L} \rho^{n} .
$$

Let $r, s$ be in $\{1, \ldots, k\}$ and let $F, H$ be measurable functions defined on $[-L, L]$. The application $T_{r} H$ (defined in (10) belongs to $V_{\alpha}(\Omega)$ if and only if $H$ is in $L^{\infty}([-L, L], m)$ and satisfies

$$
\sup _{0<\delta<\varepsilon_{0} \gamma^{1-r}} \delta^{-\alpha} \int_{[-L, L]} \operatorname{Osc}(H,] x-\delta, x+\delta[\cap[-L, L]) d x<+\infty .
$$

One then has

$$
\begin{aligned}
& \left\|T_{r} H\right\|_{\alpha, L}=(2 L)^{k-1} \gamma^{k(k-1) / 2}\|H\|_{L_{m}^{1}([-L, L])} \\
& +(2 L)^{k-1} \gamma^{(k(k-1) / 2)-\alpha(r-1)} \sup _{0<\delta<\varepsilon_{0} \gamma^{1-r}} \delta^{-\alpha} \int_{[-L, L]} \operatorname{Osc}(H,] x-\delta, x+\delta[\cap[-L, L]) d x \\
& +2^{2 k} L^{k-1}\left(\frac{1-\gamma^{k}}{1-\gamma}\right)^{k-1} \varepsilon_{0}^{1-\alpha}\|H\|_{L_{m}^{\infty}([-L, L])} .
\end{aligned}
$$

Consequently, if $H$ satisfies these conditions and if $F$ is such that $T_{s} F$ belongs to $L_{\mu}^{1}(\Omega)$, for example if $F$ is measurable and bounded on $[-L, L]$, one gets the second statement of Lemma 4

$$
\begin{aligned}
& \left|E\left(F\left(X_{n \times L^{\prime}+s-1}\right) H\left(X_{r-1}\right)\right)-\sum_{j=1}^{\operatorname{dim}\left(E_{1}\right)} \sum_{l=0}^{L_{j}-1} \mu\left(W_{j, l}\right) \mu_{j, l}\left(T_{s} F\right) \mu_{j, l}\left(T_{r} H\right)\right| \leq C\left\|T_{s} F\right\|_{L_{\mu}^{1}} \\
& \left((2 L)^{k-1} \gamma^{k(k-1) / 2}\|H\|_{L_{m}^{1}([-L, L])}\right. \\
& +(2 L)^{k-1} \gamma^{(k(k-1) / 2)-\alpha(r-1)} \sup _{0<\delta<\varepsilon_{0} \gamma^{1-r}} \delta^{-\alpha} \int_{[-L, L]} \operatorname{Osc}(H,] x-\delta, x+\delta[\cap[-L, L]) d x \\
& \left.+2^{2 k} L^{k-1}\left(\frac{1-\gamma^{k}}{1-\gamma}\right)^{k-1} \varepsilon_{0}^{1-\alpha}\|H\|_{L_{m}^{\infty}([-L, L])}\right) \rho^{n} .
\end{aligned}
$$

In particular, if $H$ is the indicator function of an interval and $F$, that of a Borel set, we obtain the first assertion of Lemma 4.

\section{A nonlinear example}

Set $\Omega=[-L, L]^{k}$. We can state the result : 
Lemma 8 Let $A>1$ and $M>0$ be real numbers. Let $\alpha_{0}, a_{1}, \ldots a_{k}, b_{1}$ be real positive numbers, with $a_{1}>0$. Set

$$
\psi(x)=\alpha_{0}+\left(\sum_{i=1}^{k} a_{i} x_{i}^{2}\right)+b_{1} x_{1} .
$$

If the following conditions are fullfilled,

$$
\begin{array}{r}
4 \alpha_{0} a_{1}=b_{1}^{2} ; \\
-2 a_{1} L+b_{1} \geq 2 A \sqrt{\frac{1}{4 a_{1}}\left(-2 a_{1} L+b_{1}\right)^{2}+\sum_{i=2}^{k} a_{i} L^{2}}>0 ; \\
\forall i \in[2, k] \cap \mathbb{N}, \sqrt{a_{1}} \sqrt{a_{i}} \leq 2 M,
\end{array}
$$

the application $\psi$ is positive on $\Omega$ and $\varphi_{0}=\sqrt{\psi}$ is well defined and $C^{\infty}$ on an open neighbourhood of $\Omega$. Moreover $\varphi_{0}=\sqrt{\psi}$ satisfies the inequalities

$$
\forall x \in \Omega,\left|\frac{\partial \varphi_{0}}{\partial x_{1}}(x)\right| \geq A, \quad \forall i \in[2, k] \cap \mathbb{N},\left|\frac{\partial \varphi_{0}}{\partial x_{1}}(x) \frac{\partial \varphi_{0}}{\partial x_{i}}(x)\right| \leq M .
$$

Proof: Thanks to (3), one gets $\psi(x)=\frac{1}{4 a_{1}}\left(2 a_{1} x_{1}+b_{1}\right)^{2}+\sum_{i=2}^{k} a_{i} x_{i}^{2}$. Hence $\psi(x) \geq \frac{1}{4 a_{1}}\left(2 a_{1} x_{1}+\right.$ $\left.b_{1}\right)^{2}$ and, since $2 a_{1} x_{1}+b_{1} \geq-2 a_{1} L+b_{1}>0$ sur [ $\left.-L, L\right]$ according to (4), $\psi$ is positive on $\Omega$. Therefore $\varphi_{0}=\sqrt{\psi}$ is well defined and $C^{\infty}$ on an open neighbourhood of $\Omega$. One checks that

$$
\frac{\partial \varphi_{0}}{\partial x_{1}}(x)=\frac{2 a_{1} x_{1}+b_{1}}{2 \sqrt{\psi(x)}} \geq \frac{2 a_{1} x_{1}+b_{1}}{2 \sqrt{\psi\left(x_{1}, L, \ldots, L\right)}} .
$$

Denoting by $g=g\left(x_{1}\right)$ the function appearing in the right side above, one sees that $g^{\prime}$ has the sign of $2 a_{1} \sum_{i=2}^{k} a_{i} L^{2}$, which means that $g$ is an increasing function. To obtain the desired condition about $\frac{\partial \varphi_{0}}{\partial x_{1}}$, it suffices that $g(-L) \geq A$, which is a consequence of (4).

One has

$$
\left|\frac{\partial \varphi_{0}}{\partial x_{1}}(x) \frac{\partial \varphi_{0}}{\partial x_{i}}(x)\right|=\frac{a_{i}\left|x_{i}\right|\left(2 a_{1} x_{1}+b_{1}\right)}{2 \psi(x)} \leq \frac{a_{i}\left|x_{i}\right|\left(2 a_{1} x_{1}+b_{1}\right)}{2 \psi\left(x_{1}, 0, \ldots, 0, x_{i}, 0, \ldots, 0\right)} .
$$

This can be written as

$$
\left|\frac{\partial \varphi_{0}}{\partial x_{1}}(x) \frac{\partial \varphi_{0}}{\partial x_{i}}(x)\right| \leq \sqrt{a_{i} a_{1}} \frac{\left(\sqrt{a_{i}}\left|x_{i}\right|\right)\left(\sqrt{a_{1}} x_{1}+\frac{b_{1}}{2 \sqrt{a_{1}}}\right)}{\left(\sqrt{a_{1}} x_{1}+\frac{b_{1}}{2 \sqrt{a_{1}}}\right)^{2}+\left(\left(\sqrt{a_{1}} x_{1}\right)^{2}\right.},
$$

and it is easy to see that it is smaller than $\frac{\sqrt{a_{i} a_{1}}}{2}$, hence smaller than $M$ according to (5).

Lemma 9 Let $A>1$ and $M>0$ be real numbers. Let $a_{1}$ and $b_{1}$ be such that

$$
\begin{array}{r}
a_{1} \geq 2 A^{2}, \\
b_{1} \geq 4 L M \sqrt{k-1}+2 a_{1} L .
\end{array}
$$

If we set $4 \alpha_{0} a_{1}=b_{1}^{2}$ (3) and impose that, for all $i$ between 2 and $k, \sqrt{a_{1}} \sqrt{a_{i}} \leq 2 M$ (5), the system of Lemma 8 is satisfied.

Proof

According to (18), $b_{1}-2 a_{1} L \geq 4 L M \sqrt{k-1}>0$ and then $\sqrt{\frac{1}{4 a_{1}}\left(-2 a_{1} L+b_{1}\right)^{2}+\sum_{i=2}^{k} a_{i} L^{2}}>0$. Moreover, using (5), one gets :

$$
2 A \sqrt{\frac{1}{4 a_{1}}\left(-2 a_{1} L+b_{1}\right)^{2}+\sum_{i=2}^{k} a_{i} L^{2}} \leq 2 A \sqrt{\frac{1}{4 a_{1}}\left(-2 a_{1} L+b_{1}\right)^{2}+(k-1) L^{2} \frac{4 M^{2}}{a_{1}}} .
$$


This last expression is smaller than $-2 a_{1} L+b_{1}$ if and only if

$$
\left(-2 a_{1} L+b_{1}\right)^{2} \geq \frac{4 A^{2}}{4 a_{1}}\left(-2 a_{1} L+b_{1}\right)^{2}+(k-1) 4 A^{2} L^{2} \frac{4 M^{2}}{a_{1}},
$$

or equivalently

$$
a_{1}\left(-2 a_{1} L+b_{1}\right)^{2} \geq A^{2}\left(-2 a_{1} L+b_{1}\right)^{2}+(k-1) 16 A^{2} M^{2} L^{2} .
$$

But according to (7) and (8),

$$
\begin{aligned}
& a_{1}\left(-2 a_{1} L+b_{1}\right)^{2}-A^{2}\left(-2 a_{1} L+b_{1}\right)^{2}-(k-1) 16 A^{2} M^{2} L^{2} \\
& \quad \geq 2 A^{2}\left(-2 a_{1} L+b_{1}\right)^{2}-A^{2}\left(-2 a_{1} L+b_{1}\right)^{2}-(k-1) 16 A^{2} M^{2} L^{2} \\
& \quad \geq A^{2}\left(\left(-2 a_{1} L+b_{1}\right)^{2}-(k-1) 16 M^{2} L^{2}\right) \geq 0 .
\end{aligned}
$$

We assume that the conditions of Lemma 9 are satisfied.

For $\ell \in[-L, L[$ and $p \in \mathbb{Z}$, set

$$
\varphi_{\ell, p}(x)=\ell+\sqrt{\psi(x)}+2 p L .
$$

One defines the application $\varphi$ almost everywhere on $\Omega$, by

$$
\varphi(x)=\varphi_{\ell,-p}(x) \quad \text { si } \quad \varphi_{\ell, 0}(x) \in[2 p L-L, 2 p L+L[.
$$

Since $\varphi_{\ell,-p}(x)=\varphi_{0}$ up to an additive constant, the conditions (6) concerning the partial derivatives are fullfilled.

Let us specify the open sets. For $p \in \mathbb{Z}$, set

$$
\mathcal{O}_{p}=\{x \in]-L, L\left[^{k}: \varphi_{\ell, 0}(x) \in\right] 2 p L-L, 2 p L+L[\} .
$$

One sees that, for $p \leq-1, \mathcal{O}_{p}$ is empty and that, otherwise,

$$
\begin{aligned}
& \mathcal{O}_{0}=\{x \in]-L, L\left[^{k}: \psi(x)<(L-\ell)^{2}\right\}, \\
& \mathcal{O}_{p}=\{x \in]-L, L\left[^{k}:((2 p-1) L-\ell)^{2}<\psi(x)<((2 p+1) L-\ell)^{2}\right\}, \quad p \geq 1 .
\end{aligned}
$$

The sets $\mathcal{O}_{p}$ are open and may be empty. We would specify which ones are empty if we really wanted to give an explicit expression of the Frobenius-Perron operator.

Put $\mathcal{S}_{p}=\left\{x \in \mathbb{R}^{k}: \psi(x)=((2 p-1) L-\ell)^{2}\right\}$.

If $\mathcal{S}_{p} \cap \Omega$ is not empty, $\frac{\partial \psi}{\partial x_{1}}(x)>0$ is valid for every point of $\mathcal{S}_{p} \cap \Omega$ according to (44), so $x_{1}$ can be considered, locally, as a $C^{\infty}$ function of the other $x_{i}$ and $\mathcal{S}_{p} \cap \Omega$ is a finite union of $C^{\infty}$ submanifolds. The edges of $\Omega$ are parts of hyperplanes and so are $C^{\infty}$ too. A submanifold $\mathcal{S}_{p}$ crosses at most $k$ hyperplanes, which implies that the maximal crossing number, $Y$, is smaller than $k+1$.

The geometrical condition, under its simple form, is satisfied. Indeed, let $U=\left(u_{1}, u_{2}, \ldots, u_{k}\right)$ and $V=\left(v_{1}, u_{2}, \ldots, u_{k}\right)$ be two points of the same set $\mathcal{O}_{p}$. On $[-L, L]^{k}, \frac{\partial \psi}{\partial x_{1}}(x)>0$, according to (4). Hence, for $t \in[0,1]$, if one assumes that $-L<u_{1}<v_{1}<L$,

$$
\psi(U) \leq \psi(t U+(1-t) V) \leq \psi(V)
$$

since the only coordinate that changes is the first one. Therefore $\psi(t U+(1-t) V)$ is in the same interval (]$((2 p-1) L-\ell)^{2},((2 p+1) L-\ell)^{2}$ [if $\left.p \geq 1\right)$ as $\psi(U)$ and $\psi(V)$. Consequently, $t U+(1-t) V$ is in $\mathcal{O}_{p}$. 


\section{Références}

[AFLV] ALVES José F., FREITAS Jorge M., LUZZATTO Stefano, VAIENTI Sandro, From rates of mixing to recurrence times via large deviations, Advances in Mathematics, 228 (2011), n 2 1203-1236.

[CE] COLLET Pierre, ECKMANN Jean-Pierre, Concepts and results in chaotic dynamics : a short course. Theoretical and Mathematical Physics. Springer-Verlag, Berlin (2006).

[GO] GOUËZEL Sébastien, Sharp polynomial estimates for the decay of correlations, Israel Journal of Mathematics Vol. 139 (2004), 29-65.

[GU] GUÉGAN Dominique, Séries chronologiques non linéaires à temps discret, Economica (1994)

[HK] HOFBAUER Franz, KELLER Gerhard, Ergodic properties of invariant measures for piecewise monotonic transformations, Mathematische Zeitschrift 180 (1982), 119-140.

[ITM] IONESCU TULCEA C.T., MARINESCU G., Théorie ergodique pour des classes d'opérations non complètement continues, Annals of Mathematics Vol. 52, n2 (1950), 140-147.

[JMN] JAGER Lisette, MAES Jules, NINET Alain, Exponential decay of correlations for a realvalued dynamical system embedded in $\mathbb{R}^{2}$. Comptes rendus - Mathématique Vol. 353, n11 (2015), 1041-1045.

[LM] LASOTA Andrzej, MACKEY Michael C., Chaos, fractals and noise : stochastic aspects of dynamics, Springer Verlag, New York (1998)

[LIV] LIVERANI Carlangelo, Multidimensional expanding maps with singularities : a pedestrian approach, Ergodic Theory and Dynamical Systems Vol. 33, n1 (2013), 168-182.

[SAU] SAUSSOL Benoît, Absolutely continuous invariant measures for multidimensional expanding maps, Israel Journal of Mathematics 116 (2000), 223-248.

[TON1] TONG Howell, Nonlinear time series. A dynamical system approach. With an appendix by K. S. Chan. Oxford Statistical Science Series, 6. Oxford Science Publications. The Clarendon Press, Oxford University Press, New York (1990).

[TON2] TONG Howel, Nonlinear time series analysis since 1990 : some personal reflections. Acta Math. Appl. Sin. Engl. Ser. 18 (2002), no. 2, 177-184.

[YOU] YOUNG Lai-Sang, Recurrence times and rates of mixing, Israel Journal of Mathematics 110 (1999), 153-188. 